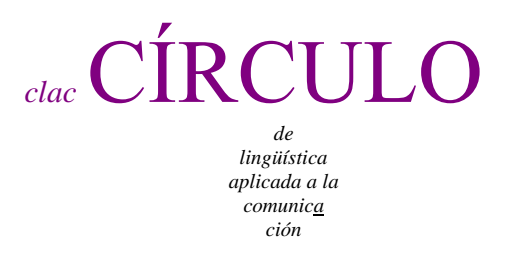

$48 / 2011$

\title{
ALTERNANCIA PREPOSICIONAL EN LOS COMPLEMENTOS \\ PREPOSICIONALES REGIDOS EN ESPAÑOL: APROXIMACIÓN SUBLÉXICA
}

Zoltan Zato

Universidad Autónoma de Madrid

zoltan zato en estudiante uam es

Resumen

En este trabajo se examina el comportamiento de las preposiciones de los complementos preposicionales regidos del español. En el primer apartado, se definen los términos preposición y complemento preposicional regido; en el segundo, se exponen algunos argumentos a favor del carácter léxico y eventivo de la preposición; en el tercero, se explica por qué el modelo del lexicón generativo de Pustejovsky es apropiado para el análisis de las preposiciones; en el cuarto, se estudia la sintaxis de los complementos preposicionales regidos; en el quinto, se muestran determinadas estructuras de qualia correspondientes al significado de varias preposiciones, y por último, en el sexto, se analizan algunas alternancias preposicionales en términos de concordancia de los rasgos semántico-aspectuales de la preposición en relación con los del verbo que la selecciona.

Palabras clave: preposición, (sub)evento, infraespecificación, concordancia de rasgos.

Zato, Zoltan. 2011.

Alternancia preposicional en los complementos preposicionales regidos en español: Aproximación subléxica.

Círculo de Lingüística Aplicada a la Comunicación 48, 41-76.

http://www.ucm.es/info/circulo/no48/zato.pdf

(C) 2011 Zoltan Zato

Círculo de Lingüística Aplicada a la Comunicación (clac)

Universidad Complutense de Madrid. ISSN 1576-4737. http://www.ucm.es/info/circulo http://dx.doi.org/10.5209/rev_CLAC.2011.v48.39031 


\begin{abstract}
Prepositional alternation in governed prepositional objects in Spanish: A sublexical approach

This paper examines the behavior of the preposition when using governed prepositional objects in Spanish. The first section defines the terms preposition and governed prepositional object; the second shows several arguments in favor of the lexical and eventive nature of the preposition; the third explains why Pustejovsky's Generative Lexicon model is appropriate for prepositional analysis; the fourth studies the syntax of the prepositional objects; the fifth shows certain qualia structures corresponding to the meaning of several prepositions; finally, the sixth analyzes several prepositional alternations in terms of the agreement of the semantical-aspectual features of the preposition in relation to the features of the verb that selects it.
\end{abstract}

Key words: preposition, (sub)event, underspecification, agreement of features.

Índice

1. Preposición y complemento preposicional regido 43

2. La preposición: categoría léxica y eventiva 44

3. La teoría del lexicón generativo 47

3.1. La estructura eventiva 48

3.2. La estructura de qualia 51

4. Sintaxis de los CPR 52

5. Significado de las preposiciones 54

5.1. Las preposiciones $a$, hacia y hasta 54

5.2. Las preposiciones de y desde 59

5.3. La preposición con 60

5.4. La preposición en 61

6. Análisis de algunos CPR 62

6.1. Atar 62

6.2. Añadir 63

6.3. Poner y llevar 64

6.4. Entrar 66

6.5. Renunciar 67

6.6. Enfrentarse 69

6.7. Cuidar 70

7. Conclusiones 71

Referencias bibliográficas 72 


\section{Preposición y complemento preposicional regido}

En las últimas décadas se ha escrito mucho acerca del intrincado mundo de las preposiciones y su relación con los complementos preposicionales regidos $(\mathrm{CPR})^{1}$. No me ocuparé aquí de repasar las diversas propuestas, sino que formularé brevemente una definición para cada uno de los términos en cuestión. En cuanto a la preposición, puede definirse como la categoría eventiva que no experimenta flexión de tiempo o aspecto, en contraposición al verbo, que sería la categoría eventiva con flexión de tiempo y aspecto. $^{2}$

Por otra parte, los CPR, siguiendo a Bosque (1983: 147) -y como su propia denominación indica一, son «sintagmas preposicionales regidos por el verbo». ${ }^{3}$ De esta definición se desprende que se consideran CPR los sintagmas preposicionales (SP) argumentales con independencia de que, por ejemplo, algunos verbos permitan su elisión o no: Me acuerdo (de ellos); El libro versa *(sobre economía), de que alternen dos o más preposiciones o no: Hablan $\{$ de/sobre $\}$ Madrid; Provienen $\left\{\mathrm{de} /{ }^{*} a / *^{*} e n / *^{* . . .}\right\}$ Madrid, o de que seleccionen una preposición con carga léxica o no: Se alejaron de aquel extraño lugar; La tesis consta de varios capítulos. ${ }^{4}$ Ello significa que en este

\footnotetext{
${ }^{1}$ Este artículo está inspirado en la tesina de fin de máster Las preposiciones del español: una aproximación subléxica, presentada el 11 de octubre de 2010 en la Universidad Autónoma de Madrid. Debo decir que no habría sido posible sin la ayuda de mi directora de tesis Elena de Miguel, a quien doy las gracias en primer lugar por su intenso trabajo y por la confianza que ha puesto en mí desde siempre. Asimismo, estoy enormemente agradecido a Cristina García, Matías Jaque y Víctor Lara por sus observaciones y por las ganas que han puesto en corregirlo y mejorarlo.
}

\footnotetext{
${ }^{2}$ Svenonius (2004) arguye que las preposiciones no experimentan flexión de tiempo precisamente porque carecen de argumento eventivo. Sin embargo, como se tratará de argumentarse en el $\S 2$, hay datos que parecen indicar que, en realidad, la preposición sí es una categoría eventiva.

${ }^{3}$ En el $\S 4$ se intenta demostrar que los CPR y los adjuntos ocupan distintas posiciones sintácticas, lo que justificaría la discriminación del CPR respecto al adjunto y, por ende, la acuñación de un término propio con el que diferenciarlo.

${ }^{4}$ Son varias las propuestas, sobre todo de corte funcionalista, con las que se han tratado de justificar distintas clases de CPR, o suplementos, según la terminología de esta corriente. Así, desde esta perspectiva, por un lado, quizá la clasificación más conocida sea la de Alarcos (1990), que distingue entre
} 
artículo no se hacen distinciones entre clases o tipos de CPR, ya que se trata de un asunto tangencial al análisis que aquí se propone. Hechas, pues, estas consideraciones, podemos pasar a examinar la hipótesis que se plantea en este artículo.

\section{La preposición: categoría léxica y eventiva}

A lo largo de este artículo se tratará de argumentar que el régimen preposicional puede explicarse sobre la base de un proceso de concordancia de rasgos semántico-aspectuales entre el verbo y la preposición. Este trabajo no va a interesarse, pues, por el origen o la evolución del régimen preposicional de un determinado verbo, sino que busca una explicación en términos de un modelo formal y asume como supuesto fundamental que esa explicación puede hallarse en la existencia de unos rasgos en el significado mínimo de la preposición regida, que han de concordar con los del verbo rector. Dicha concordancia de rasgos solo se explica por el hecho de que las preposiciones, incluidas las de los $\mathrm{CPR},{ }^{5}$ tienen significado léxico y predicación propia.

suplemento propio, indirecto, inherente o adverbial y atributivo. Por otro, Martínez García (1986) diferencia dos grandes grupos de verbos dependiendo de si pueden omitir o no el suplemento: verbos de rección subordinada, que sí pueden omitirlo, y de rección solidaria, que no.

Desde un punto de vista generativista, es notoria la clasificación de Demonte (1991), quien también diferencia dos grandes grupos de verbos —innominados- en función de si pueden elidir o no su CPR, y propone para cada uno de ellos una estructura sintáctica distinta. Por su parte, Neeleman (1997) discrimina entre SP argumentos, seleccionados por el verbo y que reciben directamente de este su papel temático, y complementos, seleccionados por el verbo pero que no reciben papel temático directamente de este (v. la nota 5). No entro a evaluar las propuestas de todos estos autores, ya que es una cuestión que excede los objetivos de este artículo.

${ }^{5}$ Sin embargo, parece que hay algunos verbos, como depender (de) o consistir (en), que seleccionan una preposición sobre la cual resulta muy difícil postular un mínimo significado nocional. Según Neeleman (1997), en estos casos la preposición se incorpora al verbo para crear, de este modo, un predicado complejo, que es el que asigna papel temático a su argumento. Por ejemplo, en John has always believed in Bill's honesty, el verbo (believe) y la preposición (in) asignan conjuntamente papel temático al constituyente Bill's honesty. 
En Chomsky (1970) se sientan las bases para formular una teoría sintáctica aplicable a las categorías léxicas — que cristalizará a breve término en la teoría de la X con barra一, pero todavía no se incluyen las preposiciones dentro de este grupo. Es a partir de Chomsky (1981) cuando se diferencian dos clases de preposiciones: las léxicas, que tienen significado nocional, asignan papel temático $\mathrm{y}$, por tanto, seleccionan argumentos, y las funcionales, que tienen significado gramatical y no asignan papel temático aunque sí caso. De hecho, una misma preposición puede ser unas veces léxica y otras, funcional. Así, la preposición de en Juan se alejó de la casa no equivale a la preposición de en La llegada de la reina. En concreto, esta última carece de significado nocional, frente a la del primer ejemplo, que aporta un claro significado de 'origen'. Así pues, considero que la oposición léxico/funcional es de carácter semántico: las categorías léxicas «remiten a realidades externas al sistema gramatical», mientras que las funcionales «se definen en función de los conceptos propios de la gramática» (Bosque y Gutiérrez-Rexach, 2009: 108).

Considero importante realizar esta aclaración, porque existen otros trabajos en los que la distinción léxico/funcional se establece sobre la base de criterios sintácticos, como en Baker (2003), Svenonius (2004, 2010) o Cinque (2010), entre otros. Como ya se ha dicho arriba, en este trabajo se considera que la oposición entre lo léxico y lo funcional es semántica y no se tienen en consideración, pues, estas propuestas. Asimismo, por falta de espacio no puedo detenerme en argumentar el carácter léxico de las preposiciones; para ello el lector interesado puede consultar, por ejemplo, Bosque (1989) o la RAE-AALE (2009), y sobre todo Horno (2002), que dedica una sección concreta a refutar las pruebas utilizadas tradicionalmente en sentido contrario.

Por otra parte, el hecho de que la preposición se caracterice por ser una categoría léxica no implica que la intensión de su significado sea alta, sino todo lo contrario. Por ejemplo, dentro del paradigma de los verbos, hay algunos cuya intensión es alta, como alunizar, y otros cuya intensión es más bien baja, como hacer, el cual es, en consecuencia, altamente polisémico. Todas las preposiciones se asemejan a este último verbo; son, pues, categorías de naturaleza soporte o ligera, lo que les permite aparecer en multitud de contextos. En los contrastes Juan hizo \{volteretas/un castillo de arena\} es el complemento directo (CD) el que dota de carga semántica a la oración, dado que el 
verbo solamente expresa la idea de 'acción'; de modo análogo, en los contrastes Juan trabaja con \{María/el martillo\}, es el término de la preposición el que determina si el complemento expresa compañía o instrumento, ya que la preposición solo denota 'concomitancia' entre el sujeto y el objeto (v. el § 5.3).

Además de tener naturaleza léxica, la preposición es una categoría eventiva, es decir, una categoría con aspecto. Son muchos los trabajos recientes que atribuyen a la preposición una naturaleza aspectual y existen varias pruebas formales que así lo demuestran. A continuación reseñaré tres: en primer lugar, las preposiciones pueden constituir una construcción absoluta que encierre, al igual que los verbos, una cláusula mínima, tanto en adjuntos, como en Nos escuchaba con los ojos cerrados (cit. por Hernanz y Suñer, 1999: 2548), como en CPR, como en Piensa en su novio ahogado (cit. por Bosque y Gutiérrez-Rexach, 2009: 427). En segundo lugar, a diferencia de los nombres y al igual que los verbos, las preposiciones pueden complementar a los verbos de percepción, que, de acuerdo con la propuesta de Maienborn (2005), permiten identificar los eventos, ya que estos deben poder percibirse: Vimos a Juan llorar; Vimos a Juan *(en) su casa. Por último, la tercera prueba, que podemos encontrar en Horno (2002), está relacionada con los verbos ser y estar del español, cuya oposición se basa en el aspecto. Los nombres no pueden aparecer como complemento de estar: *Juan está profesor, pero sí de ser: Juan es profesor. Por el contrario, las preposiciones, debido a su contenido aspectual, se combinan con estar: Juan está en Madrid, y no con ser: * Juan es en Madrid. ${ }^{6}$

En Horno (2002) pueden consultarse otras pruebas de tipo comparativo acerca del

\footnotetext{
${ }^{6}$ Según lo que se acaba de exponer, la segunda y tercera pruebas sobre la naturaleza eventiva de la preposición muestran, respectivamente, que los verbos de percepción y el verbo estar solo pueden ser complementados por categorías eventivas. Sin embargo, como me hace ver Elena de Miguel, los datos revelan, contrariamente a lo afirmado, que dichos verbos se muestran incompatibles con sustantivos eventivos, como en *Le vimos la fiesta o *La solución está la guerra, lo cual nos obliga a replantearnos qué es lo que en realidad demuestran estas pruebas. A mi juicio, se puede mantener que tanto los verbos de percepción como el verbo estar exigen ser complementados por una categoría eventiva, como el verbo o la preposición, al mismo tiempo que rechazan combinarse con cualquier sustantivo, sea eventivo o no. Es decir, se puede concluir que, además de una restricción aspectual, imponen sobre su complemento una restricción categorial, responsable de su incompatibilidad con cualquier clase de sustantivo.
} 
carácter eventivo de la preposición, relacionadas con lenguas como el sueco, el húngaro, el ruso o el árabe. Más argumentos a favor de dicho carácter eventivo pueden encontrarse, por ejemplo, en Ramchand (2003), Ramchand y Svenonius (2004) o Folli y Ramchand (2005), entre otros.

En conclusión, podemos afirmar que la preposición es una categoría léxica y eventiva. Ahora bien, para poder dar cuenta de forma más o menos adecuada de la concordancia de los rasgos aspectuales entre esta y su verbo rector, es preciso que expliquemos previamente a qué nos referimos con el concepto 'aspecto', en concreto con el concepto 'aspecto léxico', a partir de un modelo que nos permita dilucidar de qué forma se combinan las palabras de acuerdo con sus propiedades léxicas.

\section{La teoría del lexicón generativo}

La teoría del lexicón generativo (TLG), desarrollada por James Pustejovsky en numerosos trabajos, algunos de los cuales se citan en la bibliografía (Pustejovsky, 1993, 1995, 1998, 2001, y Pustejovsky y Boguraev, 1993), a grandes rasgos, trata de explicar cómo se estructuran y combinan las palabras para conformar nuevos significados. Está compuesta por cuatro niveles de representación: la estructura argumental, relacionada con el papel semántico de los argumentos de un predicado; la eventiva, vinculada con el aspecto léxico; la de qualia, que codifica información de las características fundamentales de una palabra, y la de herencia léxica, que da cuenta de la relación que guarda una palabra con otras del lexicón mental (Pustejovsky, 1995).

Como hace notar De Miguel (2009), el modelo de Pustejovsky es generativo, porque concibe que el léxico está regido por un número reducido de principios y de mecanismos que se disponen de forma muy estructurada en el interior de la propia palabra, y gracias a los cuales pueden generarse innumerables sentidos. Asimismo, el modelo es composicional, porque pretende explicar los sentidos que adquiere una oración a través de la combinación de palabras. Y, por último, es lexicista, porque supone que la combinatoria de las palabras viene determinada por las propiedades léxicas de estas, es decir: el modelo descansa, en líneas generales, sobre el principio de proyección de Chomsky (1981), según el cual las propiedades del léxico se proyectan en la sintaxis. 
No es este el lugar para desarrollar la TLG, ni siquiera para resumirla de forma somera, por lo que me limitaré a explicar los niveles de la estructura eventiva (EE) y de la estructura de qualia (EQ), ${ }^{7}$ debido a la estrecha relación que guardan estos con el tema del artículo.

\subsection{La estructura eventiva}

La EE, como ya se ha adelantado, es uno de los cuatro pilares que sustentan la TLG de Pustejovsky. En ella se dispone la información relativa al aspecto léxico de los predicados. Según la RAE-AALE (2009: 1684), el aspecto informa de «la estructura interna de los eventos, es decir, de la manera en que surgen, se terminan o se repiten, pero también de si se perciben en su integridad o se muestran únicamente algunos de sus segmentos»; por consiguiente, el aspecto léxico corresponde a la información aspectual contenida en una pieza léxica predicativa. La propuesta más generalizada en torno al aspecto léxico, inspirada en Vendler (1967), recoge cuatro clases de eventos indivisibles: estados, con duración, sin dinamismo y no delimitados; actividades, con duración, con dinamismo y no delimitados; realizaciones, con duración, con dinamismo y delimitados, y logros, sin duración, con dinamismo y delimitados.

Sin embargo, en este trabajo se sigue la propuesta de Pustejovsky — con alguna matización, que será indicada enseguida-, quien en los trabajos de 1991, 1995 y sucesivos formula que el aspecto léxico es descomponible o, en otras palabras, que los eventos están integrados por fases o subeventos, y propone tres clases aspectuales: estados, procesos y transiciones. Los estados son eventos simples que no se definen en relación con otros eventos, los procesos son secuencias de eventos que identifican la misma expresión semántica y las transiciones son eventos que identifican expresiones semánticas definidas en relación con sus opuestas (Pustejovsky, 1991). Así, en un ejemplo estativo como La puerta está cerrada, se informa de un evento independiente, «la puerta cerrada»; por su parte, en el proceso María está corriendo, existe una secuencia de eventos iguales que se repiten. Por último, en la transición La puerta se

\footnotetext{
${ }^{7}$ En De Miguel (2009) se ofrece un resumen bastante exhaustivo de la teoría. Por supuesto, es explicada y desarrollada con detalle por el propio Pustejovsky en su libro de 1995, citado en la bibliografía.
} 
cerró, el verbo cerrarse implica un estado nuevo, «cerrado», definido en oposición al estado anterior, «no cerrado». Para Pustejovsky, las realizaciones y los logros de Vendler pueden subsumirse en la misma clase aspectual, ya que comparten la característica de que denotan un cambio de estado. En realidad, lo que los diferencia es la agentividad del sujeto: las realizaciones tienen un sujeto agentivo que activa el evento, como en Juan cerró la puerta, mientras que los logros tienen un sujeto no agentivo, como en La puerta se cerró. Por lo tanto, la diferencia entre un logro y una realización no es aspectual en primera instancia, lo que justifica tal clasificación.

La novedad del modelo de Pustejovsky no estriba en la justificación de las tres clases aspectuales, sino en la concepción de que los predicados se componen de fases o subeventos, es decir, de que no son indivisibles. Sin embargo, presenta algunos inconvenientes, que expongo a continuación.

Como señala De Miguel (2009), pese a que con la clasificación de Pustejovsky se pueden explicar las alternancias entre los verbos causativos y anticausativos del tipo quemar(se), romper(se), etc., no se puede dar cuenta de los logros sin variante causativa (llegar) ni de las «alternancias entre verbos con una variante puntual (La policía rodeó el edificio a las 10:00 h) y otra durativa (La policía rodea el edificio durante horas)», donde o bien la policía es agente en los dos casos o bien si no lo es en uno es precisamente en la variante durativa. En este punto, quiero introducir la propuesta de De Miguel y Fernández Lagunilla — formulada y desarrollada en De Miguel y Fernández Lagunilla (2000, 2004), Fernández Lagunilla y De Miguel (2003) y De Miguel (1999, 2004), entre otros trabajos-, quienes matizan y amplían el modelo de Pustejovsky. Estas autoras establecen todas las combinaciones posibles entre las tres clases de eventos básicos: estados, procesos y logros, lo cual las lleva a proponer ocho clases aspectuales para el español y a abrir la posibilidad de que en otras lenguas haya otro número.

En su clasificación, el evento estado es un «evento simple, con duración y sin fases» (tener, detestar), el proceso (P1) es una «secuencia de eventos idénticos, con duración y fases» (estudiar, nadar) y el logro simple (L1) es un «evento delimitado que ocurre en un punto» (explotar, llegar). Los otros cinco eventos del español serían: transición (T1): «proceso que desemboca en un punto seguido de un cambio de estado» (leer un libro, 
ver una película); logro compuesto (L2): «evento delimitado que culmina en un punto (la fase inicial) y va seguido de un estado» (marearse, ocultarse); logro compuesto (L3): «evento delimitado que culmina en un punto (la fase inicial) y va seguido de un proceso» (hervir, florecer); transición (T2): «evento delimitado que implica una transición entre dos puntos de culminación; tanto el subevento inicial como el final pueden a su vez descomponerse en dos fases» (ir(se), caer(se)), y proceso (P2): «evento de acabamiento gradual» (adelgazar, encanecer). En suma, la propuesta de De Miguel y Fernández Lagunilla rechaza desde el principio la distinción entre el logro y la realización en función de la agentividad del sujeto, otorga entidad independiente a los logros y propugna una serie de combinaciones de subeventos que modelan distintos tipos de eventos complejos:

a. Estado

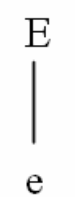

e

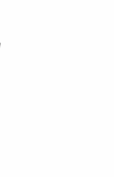

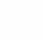

d. Logro simple (L1)

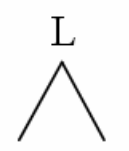

$\left(\begin{array}{ll}E_{1} & E_{2}\end{array}\right)$

g. Transición (T2)

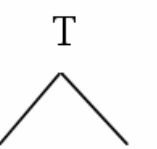

$\mathrm{L} \quad \mathrm{L}$

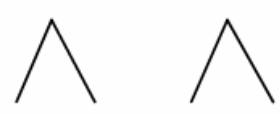

L $\quad(P) \quad L \quad(E)$ b. Proceso $(\mathrm{Pl})$

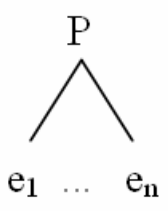

e. Logro compuesto (L2)

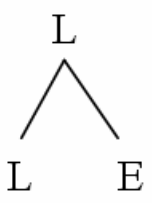

h. Proceso (P2)

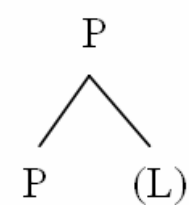

La novedad de la propuesta de De Miguel y Fernández Lagunilla no reside en las ocho clases aspectuales para el español, sino en su concepción «geométrica, articulada y recursiva» del aspecto léxico, es decir, en el hecho de que los eventos básicos puedan 
engarzarse unos con otros para formar nuevos eventos. A mi parecer, esta propuesta resulta adecuada para explicar el régimen preposicional, pues permite que podamos asociar la preposición regida con la fase verbal sobre la que proporciona información, tal y como se mostrará más adelante, en el $\S 4$.

\subsection{La estructura de qualia}

Si la propuesta de EE que acabamos de explicar apuntala nuestro análisis en el plano aspectual, ahora necesitamos de otra propuesta teórica que lo haga en el plano semántico, pues no debemos olvidar que en este artículo tratamos de explicar el régimen preposicional atendiendo a los rasgos semántico-aspectuales del verbo y la preposición, que se alojan en el interior de la palabra y que deben concertar los unos con los otros.

Según Pustejovsky (1995), el significado de las palabras está infraespecificado, es decir, las palabras contienen definiciones poco especificadas que adquieren significados más precisos cuando se combinan con otras. Asimismo, este autor postula que el significado de la palabra está codificado en la EQ, pero no se halla anclado o fijo en ella, sino que se dispone de forma ordenada y estructurada en cada uno de los cuatro qualia o roles de que se compone la palabra e interactúa y se imbrica con los demás niveles de representación, lo que quiere decir que la concepción del léxico de Pustejovsky no es estática, sino dinámica. La EQ de una palabra se compone de cuatro qualia o roles, que aluden a distintas informaciones de la entidad (evento u objeto) a la que se refieren: el rol constitutivo, que codifica información relativa a sus partes constituyentes; el rol formal, que la distingue dentro de un dominio mayor; el rol télico, que alude a su propósito y función, y el rol agentivo, que señala los factores involucrados en su origen o su producción (Pustejovsky, 1995). Estas informaciones subléxicas determinan las combinaciones de la palabra en la sintaxis; así, por ejemplo, un nombre como pista admite distintos complementos adjetivos y preposicionales que materializan esos contenidos codificados en los diferentes qualia: pista \{de hierba/de cemento/de hielo\}, quale constitutivo; pista \{rojiza/cubierta/rectangular\}, quale formal; pista \{de baile/de tenis/de patinaje\}, quale télico, y pista \{artificial/municipal/de diseño\}, quale agentivo (De Miguel, 2009). 
Por otra parte, el significado de la preposiciones, como ya se señaló en el $\S 2$, es bastante reducido, por lo que, en mi opinión, la mejor manera de describirlo es por medio de una relación de rasgos binarios. Dichos rasgos se localizan en la EQ, en concreto en los roles constitutivo y formal, en los términos que se verán en el $\S 5$.

Finalmente, antes de pasar al análisis, deben hacerse algunas consideraciones con relación a la sintaxis de las preposiciones de los adjuntos y de los CPR.

\section{Sintaxis de los CPR}

Los SP adjuntos denotan, habitualmente, un evento que complementa al evento verbal en su conjunto, mientras que los SP en calidad de CPR proporcionan o materializan información acerca de una de las fases verbales. Detengámonos en los siguientes ejemplos:

(1) a. Juan lavó el coche en el garaje.

b. Juan puso el coche en el garaje.

Aunque aparentemente estas oraciones integran los mismos constituyentes, lo cierto es que el SP en el garaje desempeña una función sintáctica diferente en cada caso: en (1a) es un adjunto de lugar, pues no forma parte de la estructura argumental del verbo, mientras que en (1b) desempeña la función de CPR, y es argumental por tanto, ya que su omisión produce una oración agramatical: Juan puso el coche *(en el garaje). ${ }^{8}$

\footnotetext{
${ }^{8}$ Hay que manejar con sumo cuidado la prueba de la elisión del complemento. Algunos complementos argumentales permiten su elisión, como en Juan come $\phi$ a las 15:00 h, donde se ha eliminado el CD, por lo que no puede asegurarse que los complementos seleccionados por el verbo hayan de explicitarse siempre. Sin embargo, si el complemento en cuestión no puede elidirse, entonces sí puede afirmarse que es un argumento del verbo, como sucede con el SP del verbo poner. En conclusión: si el complemento se puede elidir, este podrá ser argumental o no, mientras que si no permite su supresión, será argumental con toda seguridad.
}

Soy consciente de que existen, con todo, algunos casos en los que no puede elidirse un adjunto, como en Juan come *(a las 15:00 h), La modelo viste *(adecuadamente) o El puente fue construido *(\{por los romanos/en el siglo I\}), que podrían considerarse contraejemplos a la explicación que acabo de mencionar 
Además, en la primera secuencia el SP aporta información acerca del lugar donde trascurre la acción de lavar el coche, mientras que en la segunda indica la ubicación final del CD. En virtud de estos datos, debe proponerse un análisis sintáctico que refleje que el CPR ocupa una posición sintáctica diferente de la del adjunto, como trata de mostrarse a continuación con los siguientes diagramas arbóreos:

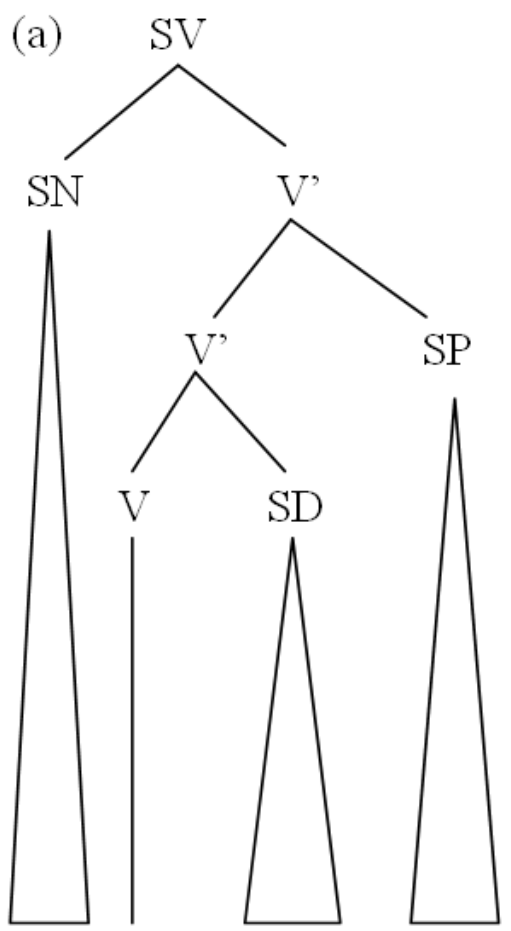

Juan lavó el coche en el garaje

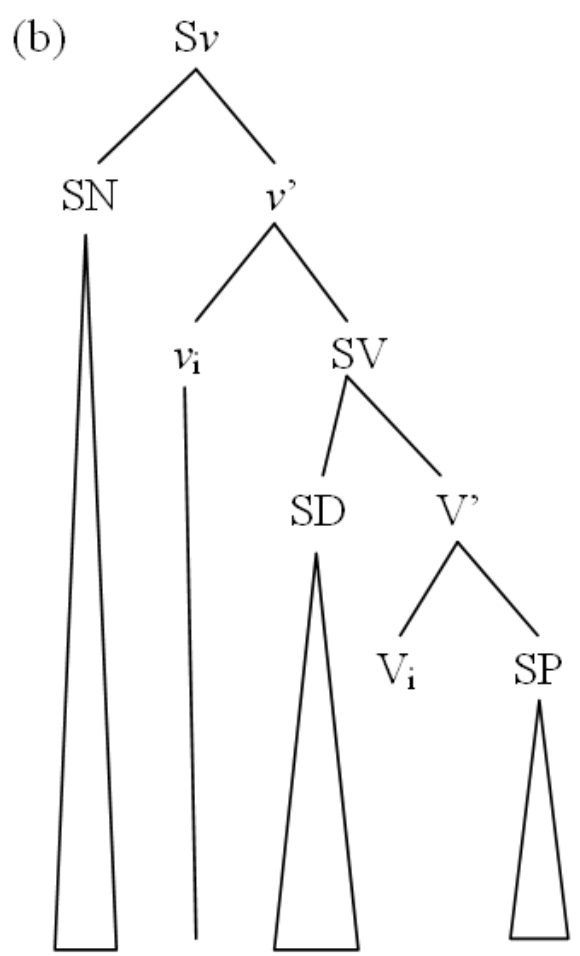

Juan puso el coche en el garaje

Como se aprecia en el diagrama (a), el SP se anexa al nudo V', en una posición de adjunto, mientras que en el diagrama (b), que está basado en la hipótesis del sintagma verbal escindido de Larson (1988), el mismo SP se adjunta directamente a V, lo que explica su condición de complemento argumental. Así pues, la acuñación de un término para el CPR y su distinción, por consiguiente, de los adjuntos se funda en dos argumentos: en primer lugar, a diferencia de los adjuntos, se trata de un complemento

sobre la prueba de la elisión. Sin embargo, creo que estos casos se explican por razones de tipo semántico más que sintáctico: a mi juicio, la obligada aparición de los adjuntos se debe a que son necesarios para que en la oración se predique algo. No es este el momento de extenderse más; para una explicación en esta línea a propósito de la elisión del sintagma-por en las pasivas con estar, consúltese De Miguel (2004). 
exigido por el verbo, en el sentido de que está subcategorizado, y, en segundo lugar, su posición configuracional es distinta: mientras que el CPR se hermana con el verbo, el adjunto se anexa al nudo V', lo cual ocasiona que el mismo SP aporte un significado distinto dependiendo de su emplazamiento sintáctico, como se ha señalado arriba.

Una vez que hemos explicado más o menos «qué supone ser CPR», podemos pasar a presentar la EQ de las preposiciones sometidas a estudio. Por razones de falta de espacio, solo pasaré revista a algunas de las preposiciones del español, en concreto $a$, con, de, desde, en, hacia y hasta. Algunas de estas preposiciones son seleccionadas por los verbos examinados en el $\S 6$.

Tal y como se había indicado en el $\S 2$, a pesar de que constituyen una categoría léxica, las preposiciones albergan muy poco significado, lo que las hace proclives a aparecer en numerosos contextos. Por esta razón, resulta útil definirlas a través de rasgos significativos mínimos, contenidos en los qualia constitutivo y formal de la palabra. Dada la extensión que implicaría un análisis que justificase los significados propuestos, debo limitarme a proporcionar los resultados obtenidos en Zato (2010). He de añadir que las EQ postuladas son fundamentales para entender las alternancias preposicionales del $\S 6$, referente al análisis.

5. Significado de las preposiciones

\subsection{Las preposiciones $a$, hacia y hasta}

En Zato (2010) propuse las siguientes EQ para las preposiciones que nos atañen en este apartado:

Quale constitutivo de $a$ : +dinamismo, +orientación, +telicidad, -trayectoria

Quale constitutivo de hasta: +dinamismo, +orientación, +telicidad, +trayectoria

Quale constitutivo de hacia: +dinamismo, +orientación, -telicidad, +trayectoria,

A partir de estas EQ, podemos postular sobre qué subeventos informan estas 
preposiciones: $a$, por no denotar trayectoria pero sí orientación, puede informar sobre logros y procesos -y algunas veces, estados (finales)—; hasta, por denotar una trayectoria delimitada, informa sobre procesos seguidos de logros, y hacia, por denotar trayectoria sin delimitar, informa sobre procesos. De ello se desprende que hay preposiciones, como hasta -y más adelante veremos que también como desde-, que pueden informar sobre más de una fase verbal. Esta idea hace hincapié en que el evento verbal ha de poder desgajarse en fases y, por tanto, en que es necesario asumir una propuesta que se fundamente en esta idea, como la de Pustejovsky o la clasificación de verbos de De Miguel y Fernández Lagunilla.

Por otra parte, según Trujillo (1971), Morera (1988) u Horno (2002), entre otros autores, estas preposiciones, junto con de y desde, tendrían el rasgo de [+dinamismo]. También en Zato (2010) asocié, no sin ciertos reparos, dicho rasgo a estas preposiciones. No obstante, podríamos prescindir de él, ya que es algo discutible que en español existan preposiciones dinámicas. Por ejemplo, como se señala en la RAEAALE (2009: 2259), hoy en día persisten «algunos usos localizadores de la preposición a, como en esperar a la entrada, sentarse a la mesa, quedarse a la puerta, ponerse al sol, tumbarse a la sombra», lo que revela que la preposición a no expresa por ella misma movimiento alguno. En sentido contrario, una preposición calificada habitualmente como 'estativa', tras, puede recibir interpretación dinámica en oraciones del tipo El perro está todo el día tras la liebre, donde el verbo es estativo y, por tanto, está incapacitado para dotar de dinamismo a la oración. Nótese, además, que en los casos de los verbos de movimiento (ir, llegar, venir, etc.) no es la preposición la que aporta el dinamismo a la oración, sino el propio verbo; a este respecto, obsérvese que el verbo entrar alterna en, preposición supuestamente estativa, y $a$, supuestamente dinámica, pero la interpretación puede ser dinámica incluso con en a causa de las propiedades semánticas del verbo, como en Juan entró \{a/en\} la habitación.

Esta es una interesante discusión que debe tratarse en profundidad, pues son muchos los autores que defienden la existencia de preposiciones dinámicas; además, resulta algo precipitado hacer afirmaciones con carácter general en lugar de estudiar el significado de cada preposición por separado. En cualquier caso, para cumplir con el propósito de este trabajo no es imprescindible resolverla ahora. 
En cuanto al rasgo [ \pm télico], es posible que también debamos considerarlo no pertinente en relación con el significado de la preposición $a{ }^{9}$ En primer lugar, dicha preposición coaparece con predicados estativos (Está sentado al piano), los cuales no tienen fases y, por tanto, no pueden acotarse. Y en segundo lugar, cuando se combina con predicados dinámicos, no siempre implica que la acción esté acabada, como en Juan lanzó un papel a la papelera, donde el papel no tuvo por qué caer dentro de la papelera: Juan lanzó un papel a la papelera, pero este cayó fuera.

A propósito de esta cuestión, Fábregas (2007) postula que la preposición a denota una relación espacial en la que la figura entra en contacto con al menos un punto de los límites del fondo. ${ }^{10}$ Desde mi punto de vista, esta caracterización no resulta del todo satisfactoria, ya que a no siempre implica contacto, como en Está sentado al piano o Está tumbado al sol. Tampoco considero adecuado incluirla dentro de las preposiciones de 'cercanía' de Svenonius (2010), ${ }^{11}$ pues, además de que esta noción puede resultar algo imprecisa, en el sentido de que es difícil determinar qué significa estar cerca o no en lingüística, creo que no sería adecuado suponer que el sujeto está cerca del objeto en, por ejemplo, El apartamento mira al mar y ni mucho menos en Juan está tumbado al sol.

El problema en torno a la posible telicidad de a proviene de que en algunos ejemplos implica acción acabada, como en Juan llegó a Madrid o en Juan entró a la habitación, mientras que en otros no, como en Juan lanzó un papel a la papelera o en Juan envió una carta a María. No obstante, creo que todos ellos pueden explicarse a partir de la hipótesis de este trabajo, siempre que postulemos que el rasgo [ \pm télico] no es pertinente en relación con $a$. Los verbos con los que esta preposición implica telicidad (llegar,

\footnotetext{
${ }^{9}$ Agradezco esta observación a Matías Jaque.

${ }^{10}$ La figura es la entidad cuya localización o movimiento se define y el fondo es el marco respecto al cual se define la localización o movimiento de la figura (Talmy, 2000). En este trabajo en lugar de figura y fondo suele hablarse de sujeto y objeto, respectivamente, ya que en la mayoría de los casos son términos equivalentes.

${ }^{11}$ En Svenonius (2010) se dividen las preposiciones del inglés delimitadas (bounded) en preposiciones que implican 'interpolación', como among y between; 'contacto', como upon y against, y 'cercanía', como beside y next to.
} 
entrar) constituyen eventos acotados sin duración, por lo que a solo puede denotar la orientación del punto en el que culmina dicho evento. En cambio, los eventos en los que a no implica telicidad contienen una fase de proceso; de esta forma, la preposición puede informar bien sobre el punto en el que culmina el evento, bien sobre el proceso inicial:

a. Juan envió una carta a María ${ }^{12}$

Ambigua

b. Juan envió una carta a María que la encandiló.

Télica

c. Juan envió una carta a María, pero nunca le llegó.

Atélica

(3)

a. Juan lanzó un globo de agua a María.

Ambigua

b. Juan lanzó un globo de agua a María en la cara.

Télica

c. Juan lanzó un globo de agua a María, pero no le dio. ${ }^{13}$ Atélica

De hecho, los respectivos nominales envío y lanzamiento revelan que las trayectorias de la carta y del globo pueden ser alteradas precisamente en la fase verbal de proceso, como prueba su combinación con durante:

(4) a. Juan envió una carta a María, pero durante el envío se extravió.

b. Juan lanzó un globo de agua a María, pero durante el lanzamiento se desvió.

Por el contrario, los respectivos nominales de llegar y entrar, llegada y entrada, puesto

12 Teniendo en cuenta que con estos verbos si el CPR se refiere a una persona, se convierte automáticamente en un complemento indirecto $(\mathrm{CI})$. Ello no altera para nada nuestro análisis, ya que la preposición tiene el mismo significado cuando el SP funciona como CPR que cuando lo hace como CI. De hecho, se podría decir que el CI es un tipo de CPR que se caracteriza por que puede pronominalizarse por me, te, le, etc.

${ }^{13}$ Es posible que la aparición del clítico le deshaga la ambigüedad a favor de la interpretación télica, lo que explicaría las presumibles siguientes incongruencias semánticas: (\#)Juan le envió una carta a María, pero nunca le llegó; (\#)Juan le lanzó un globo de agua a María, pero no le dio. Esta idea ya ha sido propuesta en Campos (1999). 
que se refieren a eventos que carecen de una fase de proceso, no pueden ver interrumpida una trayectoria que no lexicalizan:

a. \#Juan llegó a Madrid, pero durante su llegada se estropeó el autobús y no acabó en Madrid.

b. \#Juan entró a la habitación, pero durante su entrada se tropezó con una piedra y se cayó antes de llegar a entrar.

Así pues, cuando el SP precedido por a informa sobre una fase de logro, implica telicidad, mientras que cuando informa sobre una fase de proceso, no. Este fenómeno es posible porque la preposición $a$ está muy infraespecificada: solo denota [+orientación] y este rasgo es compatible con logros, pero también con procesos; de hecho, como se recoge en algunos trabajos (v. g. Morimoto, 2001, Fábregas, 2007, Cano Cambronero, 2009), en español son posibles construcciones en las que el SP precedido por $a$ complementa a un verbo de proceso, como en Juan anduvo al hospital o Juan caminó al parque. $^{14}$

Por último, no debe olvidarse que la finalidad de este trabajo es proponer unas EQ que puedan dar cuenta del régimen preposicional de cualquier clase semántica de verbos. Así, aunque, con el propósito de ilustrarlo mejor, se ha explicado el comportamiento de estas preposiciones con verbos de movimiento, el análisis es aplicable a las demás clases. Por ejemplo, como se argumentará en el $\S 6.5$, renunciar selecciona una preposición con orientación, $a$, no porque tengamos que suponer un movimiento abstracto o ficticio, sino porque existe una orientación entre el primer estado — donde

\footnotetext{
${ }^{14}$ En cuanto a hacia y hasta, estas preposiciones, como se ha señalado arriba, denotan trayectoria atélica y trayectoria télica, respectivamente, como se desprende de su combinatoria con durante y en: Caminó hacia la puerta \{durante/*en\} cinco minutos; Caminó hasta la puerta \{\#durante/en\} cinco minutos. El símbolo \# de la última secuencia indica que existe una lectura gramatical (iterativa), pero que no viene al caso al no denotar atelicidad.

Por supuesto, el significado de estas preposiciones y el de las demás sometidas a estudio ( $\$ 5.2$ y ss.) merecen un estudio más amplio y detallado. Los resultados de este artículo son, por lo tanto, provisionales.
} 
no se renuncia a algo - y el segundo estado, definido en oposición al primero — donde ya se ha renunciado a algo- - . Precisamente debido a esta orientación, podemos calificar al primero de estado inicial y al segundo, de estado final.

Esta idea se formula explícitamente, por ejemplo, en Moreno Cabrera (2003), quien, inspirándose en el concepto aristotélico de 'kínesis', manifiesta que puede hablarse de movimiento siempre que lo entendamos en el sentido de que algo se halla en un estado inicial y con el desarrollo del evento cambia a un estado final. Esto es, podemos hablar de movimiento en general no solo cuando se produce un cambio de ubicación (Juan fue de Madrid a Barcelona), sino también cuando se produce la adquisición de una nueva propiedad (Juan se doctoró en química orgánica por la Universidad de Barcelona). En este trabajo, en lugar de movimiento en general, hablamos de cambio de estado, pero, al igual que Moreno Cabrera, lo entendemos en un sentido lato, en el que caben cambios de ubicación, adquisiciones de propiedades y, por supuesto, cambios de estado como tales: físicos (Rompieron la mesa) y psicológicos (Juan se preocupó).

\subsection{Las preposiciones de y desde}

Estas preposiciones se definen por las siguientes EQ:

Quale constitutivo de de: +dinamismo, +orientación, -trayectoria

Quale constitutivo de desde: +dinamismo, +orientación, +trayectoria

La orientación de estas preposiciones es diametralmente opuesta a la de las del apartado anterior, es decir: en vez de proporcionar información acerca del estado final, informan sobre su estado inicial. Así, en Vienen \{a/hacia/hasta\} Madrid, se entiende que Madrid es la ubicación final, sea alcanzada o no; por el contrario, en Vienen \{de/desde\} Madrid, esta ciudad se concibe como la ubicación inicial del sujeto. Asimismo, desde contiene un componente de trayectoria del que carece de, lo que incapacita a esta última para complementar a verbos que lexicalizan trayectoria: Lo vemos $\{d e s d e / * d e\}$ aquí; Llevamos esperando $\{$ desde/*de $\}$ las 17:00 h. 
Paralelamente a como se decía supra, las propiedades semánticas de las preposiciones de y desde nos permiten dilucidar sobre qué subeventos verbales informan: de, por denotar orientación pero no trayectoria, informa sobre subeventos iniciales, mientras que desde, por poseer ambos rasgos, informa sobre logros seguidos de procesos. La clasificación de eventos de Pustejovsky no es del todo válida para explicar el comportamiento de desde, ya que en ella no se recogen eventos compuestos por un logro seguido de un proceso. Para explicar el comportamiento de esta preposición resulta necesario acudir a la clasificación de De Miguel y Fernández Lagunilla, donde se postula una combinatoria de fases más libre.

Por otra parte, como se decía arriba respecto de $a$, estas EQ también son aplicables a los verbos en los que el cambio de estado no es de tipo locativo. Así, tanto en disuadir como en venir entendemos que se produce un cambio de estado y que en ambos casos el CPR encabezado por de informa sobre el contenido del no-estado o estado inicial: aquello que no se hace y aquel lugar en el que no se está, respectivamente.

\subsection{La preposición con}

La EQ de la preposición con es la siguiente:

Quale constitutivo de con:-dinamismo, -orientación, +concomitancia

El rasgo [+concomitancia] alude a que esta preposición expresa coincidencia de entidades, como en María viene con sus padres, donde María y sus padres coinciden en el espacio y el tiempo. Luego, del significado principal derivan los demás sentidos que puede adquirir esta preposición: 'compañía', como en el ejemplo que se acaba de citar; 'causa', como en El hierro se oxida con el agua; 'relación recíproca', como en Juan se casó con María; 'relación en un solo sentido', como en Juan se identifica con María; 'instrumento', como en Golpeó el clavo con el martillo, etc. Dado que la preposición no denota orientación por sí misma, sino que esta depende del verbo (compárese casarse 
con identificarse, por ejemplo), ${ }^{15}$ incluso se podría considerar el rasgo [-orientación] como no pertinente. ${ }^{16}$

\subsection{La preposición en}

Sobre la preposición en simplemente quiero subrayar la idea de que denota ubicación referencial sin que ni siquiera deba existir contacto entre el sujeto y el objeto, como en Te espero en la puerta (RAE-AALE, 2009). Esta idea explicaría casos como el de Los comensales se sentaron en la mesa, que es una secuencia ambigua, ya que puede significar 'sobre la mesa' o 'ante la mesa' —este último uso ha sido tachado tradicionalmente de incorrecto, aunque en el Diccionario panhispánico de dudas (2005: s. v. sentar(se)) ya se da por aceptable-.

La EQ de la preposición en es la siguiente:

Quale constitutivo de en:-dinamismo,-orientación, +ubicación referencial

Como se habrá podido observar, los datos muestran que las preposiciones pueden definirse mediante una serie de rasgos semánticos, pero no denotan un subevento en concreto. Por ejemplo, las propiedades semánticas de $a$ le permiten informar sobre un logro en Llegó a Madrid o sobre un estado en Está a la sombra. Del mismo modo, las propiedades semánticas de con le permiten informar sobre un logro en Se chocó con la pared o sobre un proceso en Soñó con María. Así, puede completarse la EQ propuesta para cada preposición con el rasgo de argumento eventivo e, pero sin especificar su clase aspectual. Por ejemplo:

\footnotetext{
${ }^{15}$ Así, una oración como Juan se casó con María implica que María se casó con Juan; en cambio, en Juan se identifica con María, María no tiene por qué identificarse con Juan.

${ }^{16}$ Como ya mencioné supra en la nota 14 , tanto los rasgos de las preposiciones como el análisis en el que estos están basados constituyen una primera aproximación y han de considerarse provisionales; en cualquier caso, para más detalles el lector puede acudir a Zato (2010).
} 


\section{EN}

Quale constitutivo:-dinamismo,-orientación, +ubicación referencial

Quale formal: $x$ en $y$; +e [(E), (P), (L), etc.],

donde $x$ es el sujeto (o figura) e $y$ el objeto (o fondo).

\section{Análisis de algunos CPR}

A continuación se presentan los análisis de unos cuantos verbos con arreglo a la propuesta del trabajo: en líneas generales, en este apartado se comprobará con datos el carácter léxico y eventivo de la preposición de los CPR y se intentará explicar el régimen preposicional en razón de la concordancia de rasgos entre el verbo y su preposición correspondiente. Al final de cada subapartado se ofrece la EE propuesta para cada verbo analizado.

\subsection{Atar}

El verbo atar alterna las preposiciones $a$, en y con, con la distribución que se muestra en los ejemplos de (6):
a. Ataron el burro $\left\{\mathrm{en} / \mathrm{a} /{ }^{*} \operatorname{con}\right\}$ la pared.
b. A: ¿Dónde ataron el burro? B: $\left\{{ }^{*} \mathrm{~A} / \mathrm{en} /{ }^{*} \operatorname{con}\right\}$ la pared.
c. Ataron el burro $\{*$ en/a/con $\}$ la mula.

En (6a), el término preposicional designa una locación, por lo que resulta extraña la aparición de la preposición con, que - pese a que denota concomitancia del sujeto con el objeto- no suele adquirir valor locativo. Esto se ve con claridad en (6b), donde, por cierto, tampoco es posible la aparición de $a$, ya que esta preposición expresa 'lugar adonde', i. e. con orientación, cuando se está preguntando por un 'lugar en donde', i. e. sin orientación. En (6c) el término preposicional designa una entidad animada, por lo que la preposición locativa carece de legitimidad para este contexto; por su parte, la 
preposición con denota coincidencia de ambas entidades. La preposición a puede aparecer tanto en (6a) como en (6c) porque solo indica o el proceso en el que se ata al burro o el punto en el que el burro pasa a estar atado, sin especificar a qué clase de dominio.

EE de atar algo $+\{$ a/en/con $\}$ :

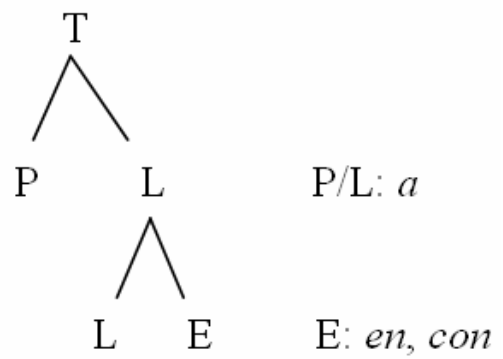

\subsection{Añadir}

El verbo añadir permite la conmutación entre en y a, como en Juan añadió azúcar \{en/a\} la leche. Si seguimos la propuesta defendida en este trabajo, con en se informa de la ubicación final, mientras que con a se informa del proceso o del punto de cambio. La preposición en tiene legitimidad en este ejemplo porque puede entenderse que la ubicación final del azúcar es la leche. De ahí se explica el contraste con Juan añadió veinte metros cuadrados $\{a / * e n\}$ su casa, donde los veinte metros cuadrados no pueden denotar una entidad localizable en la casa. Adviértase que la primera oración es equivalente a Juan echó azúcar en la leche, mientras que la segunda equivale más bien a Juan aumentó en veinte metros cuadrados su casa (cf. *Juan echó veinte metros cuadrados en su casa). ${ }^{17}$ Así las cosas, el verbo añadir pertenece a un grupo de verbos en los que es pertinente a veces expresar el sentido del movimiento y otras veces la ubicación final.

\footnotetext{
${ }^{17}$ Rechazo justificar la agramaticalidad de *Juan añadió veinte metros cuadrados en su casa por el hecho de que los veinte metros cuadrados no se hallen dentro de los límites de la casa —a diferencia del azúcar con respecto a la leche, en la otra secuencia-, dado que, como se expuso en el § 5.4, la preposición en ni siquiera exige contacto entre el sujeto y el objeto, como en Juan espera en la puerta.
} 
EE de añadir algo $+\{a / e n\}:$

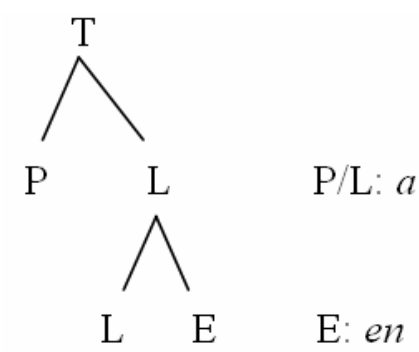

\subsection{Poner y llevar}

Los verbos poner y llevar denotan ambos movimiento, pero presentan una importante disimilitud: poner se construye con en, mientras que llevar se construye con $a$. La explicación radica en que con poner se informa sobre la ubicación final del tema, como en Puso los libros en la mesa, mientras que llevar solo da cuenta del sentido del movimiento de este, como en Llevó los libros a la mesa. Como prueba de que el CPR de llevar informa sobre el proceso o el logro y el de poner informa sobre el estado final, puede aducirse que en el primer caso el término de la preposición puede referirse a una persona, con la consecuencia de que el segmento locativo se convierte en un CI, mientras que en el segundo caso, no:

(7) a. Juan llevó los libros a la mesa.

b. Juan (le) llevó los libros a su padre.

(8) a. Juan puso los libros en la mesa.

b. *Juan puso los libros en su padre.

Por un lado, llevar permite la construcción con CI de persona porque informa sobre el punto de cambio en el que los libros pasan a estar en otro dominio, sin especificar de qué tipo. Por otro, en (8b) se muestra que una persona no puede desempeñar el papel de una locación. Este análisis puede hacerse extensible a otros verbos: por ejemplo, 
mientras que mandar o trasvasar, verbos que rigen $a$, se comportan como llevar, otros como endosar o tumbar, que rigen en, se comportan como poner:

(9) a. Juan mandó una carta a París.

b. Juan (le) mandó una carta a su madre.

(10) a. Trasvasaron el agua a Castilla la Mancha.

b. (Le) trasvasaron los poderes al emperador. ${ }^{18}$

(11) a. Juan endosó el armario en la pared.

b. *Juan endosó el armario en su hermano.

(12) a. Juan tumbó la mesa en el suelo.

b. *Juan tumbó la mesa en su hermano.

EE de llevar algo + a:

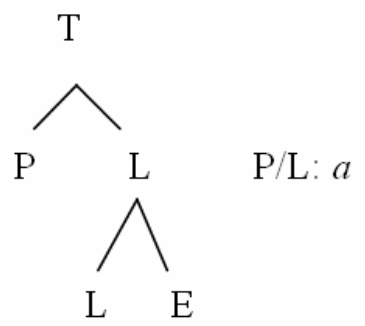

EE de poner algo + en:

L

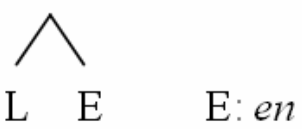

${ }^{18}$ Sobre la presencia o ausencia del clítico en esta clase de verbos, véase la nota 13. 


\subsection{Entrar}

El verbo entrar tiene una casuística variada, que intentaremos explicar con detalle. En cuanto a su EE, representa un logro compuesto (L2). Está formado por un logro porque admite la combinación con, por ejemplo, la locución adverbial de pronto; asimismo, este logro está seguido de un estado, como prueba el hecho de que pueda coaparecer con un SP precedido por durante, que denota el tiempo que se extiende el estado final:

(13) De pronto Juan entró en la habitación.

(14) Juan entró en la habitación durante unos minutos.

Ahora, detengámonos en las siguientes secuencias:

(15) a. La pelota entró $\{$ en/*a\} la habitación al ser empujada por la corriente.

b. La empresa entró \{en/*a\} quiebra en 1990.

c. La noche entró al cuarto con su frescor siempre puro (cit. por Morera, 1988: 149).

Los ejemplos de (15) ponen de manifiesto que la agentividad del sujeto está implicada de forma directa en la selección preposicional; los sujetos no agentivos, como la pelota o la empresa, no pueden combinarse con la preposición $a$. Por su parte, el ejemplo (15c) es gramatical porque el sujeto está personificado, con el objeto de conseguir un efecto retórico. Así pues, puede concluirse que para que la preposición a pueda coaparecer con el verbo entrar, el sujeto debe recibir el papel temático de agente. De este modo, cuando el sujeto es una persona, como en (16a), y la oración se construye con $a$, este es obligatoriamente un agente, mientras que cuando se construye con en, como en (16b), la oración resulta ambigua y el sujeto puede ser un agente o un tema, entendiendo por este último el 'participante que se mueve': 
(16) a. Juan entró a la habitación \{para revisar los fusibles/??al ser empujado por la corriente de agua\}.

b. Juan entró en la habitación \{para revisar los fusibles/al ser empujado por la corriente de agua\}. ${ }^{19}$

EE de entrar $+\{e n / a\}$ :

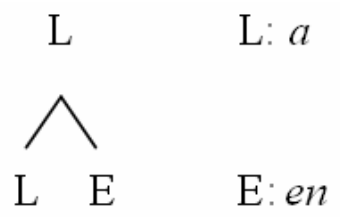

\subsection{Renunciar}

El verbo presenta una casuística interesante. Se trata de un logro simple, que implica un punto de cambio entre un estado inicial y un estado final. La presencia de la preposición $a$ es esperable, pues informa sobre el propio subevento logro:

(17) Lo cual no significa que Eltsin vaya a renunciar a que Rusia desempeñe un papel de primer orden en el Viejo Continente (La Vanguardia, 1994).

No obstante, se documentan ejemplos de régimen con preposición de:

(18) Pero eso sería traicionar a mis padres, renunciar de mis orígenes (Víctor Chamorro, El muerto resucitado, 1984).

(19) El problema más grande ha sido en relación a la convocatoria de jugadores en el sentido de que algunos han optado por renunciar de la Selección (El Salvador Hoy, 1997).

\footnotetext{
${ }^{19}$ Un estudio más profundo requeriría explicar por qué $a$ induce el papel semántico de agente, mientras que en permite el de tema. Con respecto a esto, cabe aducirse que pueden llevar $a$ tanto verbos inergativos, v. g. ir, dirigirse, como inacusativos, v. g. llegar, caer, por lo que no puede generalizarse respecto a todos los verbos que la preposición $a$ fuerce la agentividad del sujeto o viceversa.
} 
Explicar la presencia de la preposición de por medio de una supuesta analogía con el verbo renegar (de) puede ser válido pero insuficiente. Morera (1988: 209) perfila algo más esta idea y señala que «debido al carácter 'separativo' del significado léxico de este verbo [...] es perfectamente explicable que en muchas ocasiones se encuentre construido con de»; pero ello no explica por qué se produce la alternancia con a ni por qué, por ejemplo, el verbo renegar solo selecciona de. Por otra parte, no parece indicado afirmar que con renunciar «el régimen con a implica una cierta idea de "separación" o “alejamiento"», como apunta Cano Aguilar (1999: 1824), ya que supondría la asunción de un nuevo significado para la preposición $a$.

La clave se encuentra en la información aspectual. La preposición de informa sobre el estado inicial, cuya temporalidad anterior puede demostrarse por el hecho de que en los testimonios en los que aparece esta preposición se renuncia de algo que se tiene, mientras que se renuncia a algo a lo que se aspira (o también se tiene pero se ve en perspectiva). En los ejemplos (18) y (19), los sujetos renuncian de algo que tienen, como los orígenes y la selección nacional, mientras que en (17) se renuncia a una aspiración, que Rusia desempeñe un papel relevante.

Así, el hecho de que con la alternancia de las preposiciones a y de la renuncia se produzca en distintas fases del evento comporta cambios de significado, que en algunos casos pueden ser sustanciales. Por ejemplo, una oración como Juan ha renunciado al cargo es ambigua, puesto que puede interpretarse que Juan dimitió de su cargo o que rechazó una oferta; en cambio, en Juan ha renunciado del cargo, debido a la preposición de estado inicial de, solo cabe la primera interpretación. Esta misma idea da cuenta, además, de por qué renegar se construye siempre con de, pues, a diferencia de renunciar, siempre se reniega de algo que se tiene.

EE de renunciar $+\{a / d e\}:$

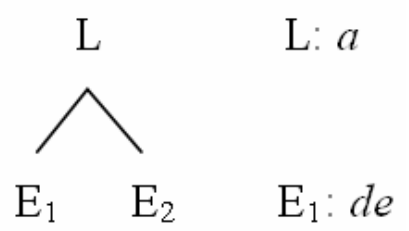




\subsection{Enfrentarse}

El verbo enfrentarse alterna a y con. Además de dicha alternancia, este verbo permite una construcción recíproca: La policía y los estudiantes se enfrentaron los unos $\{a / c o n\}$ los otros. Rodríguez García (1997: 301) señala que en una construcción «semántica recíproca», como La policía se enfrentó con los estudiantes, existe siempre una perspectiva pragmática, según la cual «uno de los co-agentes recíprocos se considera más importante y por eso se codifica como sujeto». Por otra parte, apunta que en dicha oración se sugiere «la idea de que la policía fue la responsable del incidente, aunque los jóvenes también participaron». A mi juicio, este análisis merece alguna matización.

En primer lugar, la única construcción recíproca es la primera de todas, La policía y los estudiantes se enfrentaron los unos \{a/con\} los otros, pues, como señala Bosque (1985), el concepto de 'reciprocidad' alude a una realidad sintáctica, demostrable con la inclusión del sintagma el uno P el otro (cf. * La policía se enfrentó con los estudiantes los unos con los otros). Además, puesto que ambos participantes, la policía y los estudiantes, forman parte del sujeto, esto es, reciben el papel semántico de agente, se entiende que ambos activan y controlan la acción.

En segundo lugar, la construcción no recíproca puede llevar a o con, pero solo uno de los participantes puede desempeñar la función de sujeto, con lo que, de acuerdo con el criterio temático, ${ }^{20}$ únicamente el que lo haga podrá recibir el papel semántico de agente $\mathrm{y}$, por ende, considerarse el causante del enfrentamiento, en el caso de arriba la policía. Asimismo, con $a$, preposición orientada, el sentido del enfrentamiento es único y el sujeto es exclusivamente el participante que realiza la acción de enfrentarse. En cambio, con la preposición con, cuyo significado se basa en la concomitancia entre el sujeto y el objeto, cabe la interpretación de que dicho objeto intervenga en el enfrentamiento. En suma, como en el caso del análisis de los verbos anteriores, el régimen verbal de enfrentarse también puede explicarse a partir de la concordancia de los rasgos semántico-aspectuales de la preposición y su verbo rector, sin necesidad de acudir a una hipotética perspectiva pragmática, como propone Rodríguez García.

\footnotetext{
${ }^{20}$ De acuerdo con la versión clásica del modelo chomskiano, el criterio temático es un principio que regula la proyección de las propiedades del léxico en la sintaxis. Se formula como sigue: cada argumento recibe un solo papel temático y cada papel temático se asigna a un solo argumento (Chomsky, 1981).
} 
EE de enfrentarse $+\{a, c o n\}$ :

$\mathrm{L}$<smiles>PC[Te]</smiles>

L/P: $a, c o n$

\subsection{Cuidar}

Para terminar, el verbo cuidar puede ser transitivo, y entonces significa 'tratar con cuidado', o bien puede regir la preposición de, y entonces significa más bien 'estar al cuidado':

(21) a. El caballero cruzado cuidó el libro sagrado \{durante/*en\} dos años.

b. El caballero cruzado cuidó del libro sagrado \{durante/*en\} dos años.

Las oraciones de (21) describen procesos sin delimitar, pues ambas admiten un sintagma temporal encabezado por durante, pero no por en, por lo que parece que no existe una diferencia aspectual visible. En cuanto al significado, en la primera oración se expresa que el caballero trató con cuidado el libro, mientras que en la segunda se nos informa de que el caballero estuvo al cuidado de este. La diferencia radica en que sin la preposición, el verbo se apoya únicamente en un $\mathrm{CD}$ de objeto concreto, una entidad, por lo que el cuidado se limita al estado físico de esta; en cambio, la preposición de establece una predicación y convierte el objeto en el asunto o tema sobre el que se proyecta el cuidado. $^{21}$

Obsérvese que (21a) puede parafrasearse por El caballero cruzado fue cuidadoso con el libro sagrado, mientras que el ejemplo de (21b) lo parafrasearíamos más bien por El caballero cruzado fue cuidador del libro sagrado. De hecho, en esta última oración se

\footnotetext{
${ }^{21}$ Como habrá podido advertirse, el régimen preposicional de cuidar no puede explicarse directamente a partir de la EQ propuesta para de en el $\S 5.2$, pues aquí dicha preposición no denota 'orientación', sino 'tema' o 'asunto'. Pospongo para una futura investigación un análisis que permita dar cuenta de este fenómeno y que, además, pueda aplicarse al análisis de otros verbos en los que de parece significar también 'tema' o 'asunto', como hablar, informar, etc.
} 
nos dice que el caballero estuvo al cuidado del libro independientemente de que fuera cuidadoso con él o no. Por ello, creo que el nominal cuidador deriva de cuidar 'estar al cuidado', mientras que el adjetivo cuidadoso deriva de cuidar 'tratar con cuidado', lo que demuestra que cuidar puede expresar dos significados distintos, que, según mi hipótesis, corresponden a las dos construcciones que permite dicho verbo.

Así las cosas, si el término preposicional solo puede denotar el asunto sobre el que se proyecta el cuidado, la ausencia de la preposición producirá una oración agramatical, como se aprecia en (22):

(22) El rey cuida *(de) el cumplimiento de la Constitución.

En efecto, puesto que el término preposicional se refiere exclusivamente a una predicación, i. e. algo que no puede 'tratarse con cuidado', la preposición de se hace obligatoria.

EE de cuidar + de:

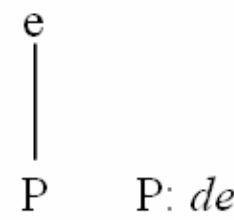

\section{Conclusiones}

En este trabajo se ha propuesto que la selección preposicional se basa en la concordancia de rasgos semántico-aspectuales entre el verbo y la preposición. Esta concordancia de rasgos es posible porque, en líneas generales, se asume que la preposición, al igual que el verbo, es una categoría léxica y eventiva.

La ventaja de esta propuesta es que nos permite tratar con una misma explicación el análisis de los casos de rección en los que hay verbos de significado dispar pero que seleccionan la misma preposición. Por ejemplo, el régimen preposicional de los verbos 
llegar y renunciar se explica a partir del mismo argumento: puesto que ambos denotan un logro, ambos seleccionan una preposición de logro, con independencia de que el primero sea un verbo de movimiento y el segundo, no.

En resumen, el propósito de este trabajo es acercarse al mundo de las preposiciones de los CPR teniendo en cuenta su carácter léxico y eventivo. Desde esta perspectiva, son muchos los caminos que se han abierto y también las cuestiones que quedan pendientes en torno al estudio de esta categoría gramatical que tanto interés despierta.

Referencias bibliográficas

Alarcos, E. (1990): «La noción de suplemento», Homenaje al profesor Francisco Marsá. Jornadas de filología, Universitat de Barcelona, 209-221.

Baker, M. (2003): Lexical categories. Verbs, Nouns and Adjectives, Cambridge, Cambridge University Press.

Bosque, I. (1983): «Dos notas sobre el concepto de "suplemento" en la gramática funcional», Dicenda, 2, 147-156.

Bosque, I. (1985): «Sobre las oraciones recíprocas en español», Revista Española de Lingüística, 15-1, 59-96.

Bosque, I. (1989): Las categorías gramaticales, Madrid, Síntesis.

Bosque, I. y Gutiérrez-Rexach, J. (2009): Fundamentos de sintaxis formal, Madrid, Akal.

Campos, H. (1999): «Transitividad e intransitividad», en Bosque, I. y Demonte, V. (coords.), Gramática descriptiva de la lengua española, Madrid, Espasa Calpe.

Cano Aguilar, R. (1999): «Los complementos de régimen verbal», en Bosque, I. y Demonte, V. (coords.), Gramática descriptiva de la lengua española, Madrid, Espasa Calpe. 
Cano Cambronero, M. ${ }^{a}$ Á. (2009): «La entrada léxica de los verbos de movimiento: los contenidos sintácticamente relevantes», ELUA, 23, 51-72.

Chomsky, N. (1970): «Remarks on nominalization», en Jacobs, R. y Rosenbaum, P. (eds.), Readings in English Transformational Grammar, Waltham, M. A. Ginn, 184-221.

Chomsky, N. (1981): Lectures on goverment and biding, Dordrecht, Foris.

Cinque, G. (2010): «Mapping spatial PPs: an introduction», en Cinque, G. y Rizzi, L. (eds.), Mapping Spatial PPs. The Cartography of Syntactic Structures, vol. 6, Nueva York, OUP.

De Miguel, E. (1999): «El aspecto léxico», en Bosque, I. y Demonte, V. (coords.), Gramática descriptiva de la lengua española, Madrid, Espasa Calpe.

De Miguel, E. (2004): «La formación de pasivas en español. Análisis en términos de la estructura de qualia y la estructura eventiva», Verba Hispanica, XII, 107-129.

De Miguel, E. (2009): «La Teoría del Lexicón Generativo», en De Miguel, E. (ed.), Panorama de la lexicología, Barcelona, Ariel.

De Miguel, E. (2000): «El operador aspectual se»,Revista Española de Lingüística, $30: 1,13-43$.

De Miguel, E. y Fernández Lagunilla, E. (2004): «Un enfoque subeventivo de la relación entre predicados secundarios y adverbios de manera», Revue Romane, 39:1, 24-44.

Demonte, V. (1991): Detrás de la palabra. Estudios de gramática del español, Madrid, Alianza Editorial.

Fábregas, A. (2007): «An Exhaustive Lexicalisation Account of Directional Complements», Tromsø Working Papers on Language \& Linguistics: Nordlyb, 34, 165-199.

Fernández Lagunilla, M. y De Miguel, E. (2003): «Adverbios de manera e información aspectual», Círculo de Lingüística Aplicada a la Comunicación 13, 3-12, 
http://www.ucm.es/info/circulo/no13/lagumigu.pdf.

Folli, R. y Ramchand G. (2005): «Prepositions and results in Italian and English: Analysis from event decomposition», en Verkuyl, H., De Swart, H. y Van Hout, A., Perspectives on Aspect, Dordrecht, 81-105.

Hernanz, M. a L. y Suñer, A. (1999): «La predicación: la predicación no copulativa. Las construcciones absolutas», en Bosque, I. y Demonte, V. (coords.), Gramática descriptiva de la lengua española, Madrid, Espasa Calpe.

Horno Chéliz, M. ${ }^{\mathrm{a}}$ del C. (2002): Lo que la preposición esconde, Zaragoza, Prensas Universitarias de Zaragoza.

Larson, R. K. (1988): «On the Double Object Construction», Linguistic Inquiry, 19, 335-391.

Martínez García, H. (1986): El suplemento en español, Madrid, Gredos.

Maienborn, C. (2005): «On the limits of the Davidsonian approach: The case of copula sentences», Theoretical Linguistics, 31, 275-316.

Moreno Cabrera, J. C. (2003): Semántica y gramática. Sucesos, papeles semánticos y relaciones sintácticas, Madrid, Antonio Machado Libros.

Morera Pérez, M. (1988): Estructura semántica del sistema preposicional del español moderno y sus campos de usos, Puerto del Rosario, Servicio de publicaciones del excmo. cabildo insular de Fuerteventura.

Morimoto, Y. (2001): Los verbos de movimiento, Madrid, Visor Libros.

Neleeman, A. (1997): «PP-complements», Natural Language and Linguistic Theory, $15,89-137$.

Pustejovsky, J. (1991): «The syntax of event structure», Cognition, 41, 47-81.

Pustejovsky, J. (1993): «Type Coertion and Lexical Selection», en Pustejovsky, J. (ed.), Semantics and the lexicon, Dordrecht, Kluwer, 73-94. 
Pustejovsky, J. (1995): The generative lexicon, Massachusetts, MIT Press.

Pustejovsky, J. (1998): «The Semantics of Lexical Underspecification», Folia Linguistica, 33, 3.4, 327-347.

Pustejovsky, J. (2001): «Type Construction and the Logic for Concepts», en P. Bouillon y F. Busa (eds.), The Syntax of Word Meaning, Cambridge, Cambridge University Press, 91-123.

Pustejovsky, J. y Boguraev, B. (1993): «Lexical knowledge representation and natural language processing», Artificial Intelligence, 63, 193-223.

RAE-AALE (2009): Nueva gramática de la lengua española, Madrid, Espasa Libros.

RAE: Banco de datos (CREA) [en línea]. Corpus de referencia del español actual. $<$ http://www.rae.es $>$ [febrero de 2011].

Ramchand, G. (2003): «First phase syntax», ms., Oxford University.

Ramchand, G. y Svenonius, P. (2004): «Prepositions and External Argument Demotion», en Solstad, T. et alii (eds.), Demoting the Agent: Passive and other Voice-related Phenomena, Universidad de Oslo, ILOS. http://www.hum.uit.no/a/svenonius/papers/RamchandSvenonius04Oslo.pdf

Rodríguez García, L. (1997): «Aplicaciones al principio de iconicidad en la extensión metafórica de la transitividad prototípica», Homenaje al Prof. J. Cantera, Universidad Complutense de Madrid.

Svenonius, P. (2004): «Adpositions, Particles, and the Arguments they Introduce», en Solstad, T. et alii (eds.), Demoting the Agent: Passive and other Voice-related Phenomena, Universidad de Oslo, ILOS.

Svenonius, P. (2010): «Spatial P in English», en Cinque, G. y Rizzi, L. (eds.), Mapping SpatialPPs. The Cartography of Syntactic Structures, vol. 6, Nueva York, OUP.

Talmy, L. (2000): «Figure and Ground in Language», Toward a Cognitive Semantics, Massachusetts, MIT Press. 
zato: alternancia preposicional 76

Trujillo, R. (1971): Notas para un estudio de las preposiciones españolas, Bogotá, Instituto Caro y Cuervo.

Vendler, Z. (1967): «Verbs and Times», Linguistics in Philosophy, Ithaca, Cornell University Press, 97-121.

Zato, Z. (2010): Las preposiciones del español: una aproximación subléxica, tesina de fin de máster, en prensa, Universidad Autónoma de Madrid.

Recibido: 21 octubre 2011

Aceptado: 20 enero 2012

Revisado: 14 febrero 2012

Publicado: 27 febrero 2012

Actualizado: 1 marzo 2012 